\title{
Análise newtoniana de um sistema mecânico com uma força dependente da velocidade e a existência de condições para a conservação de energia (Newtonian analysis of a mechanical system subjected to a velocity dependent force and the existence of conditions to energy conservation)
}

\author{
R.V. Sampaio ${ }^{1}$, R. Fracalossi² ${ }^{2}$ A.M. Oliveira ${ }^{\text {Th }}$ \\ ${ }^{1}$ Grupo de Física Aplicada, Departamento de Física, Universidade Federal do Espírito Santo, Vitória, ES, Brasil \\ ${ }^{2}$ Faculdades Integradas de Aracruz, Aracruz, ES Brasil \\ ${ }^{3}$ Grupo de Estudos Interdisciplinares em Ciências da Natureza, Guarapari, ES, Brasil \\ Recebido em 10/1/2013; Aceito em 19/7/2013; Publicado em 6/2/2014
}

\begin{abstract}
Um sistema mecânico com forças dependentes da velocidade é estudado. Mostra-se que, sob escolhas muito particulares para os termos da força resultante em cada direção, é possível obter o resultado de que a energia total do sistema se conserva, podendo ainda ser dividida em três parcelas, associadas a cada direção, e que também se comportam como uma lei de conservação para a energia individualmente.

Palavras-chave: conservação de energia, forças dependentes da velocidade, mecânica newtoniana.
\end{abstract}

A mechanical system with a velocity-dependent force is analyzed. It is shown that with very particular choices for the resulting force terms it is possible to obtain the result that the total energy of the system is conserved and that it can be divided into three groups, associated with each direction, that hold as individual conservation laws.

Keywords: energy conservation, velocity-dependent forces, newtonian mechanics.

\section{Introdução}

Desde o advento da física moderna, problemas em mecânica clássica têm perdido parte de seu prestígio devido ao fato de se tratarem de situações que, se não plenamente discutidas, ao menos já possuem seus possíveis comportamentos bem estabelecidos. Desta forma, criase uma tendência em muitas partes da física de não mais se preocupar com novos problemas em mecânica newtoniana mas de buscar entender novos sistemas cujas ferramentas matemáticas e teorias físicas sejam mais sofisticadas. Entretanto, a relativamente recente abordagem de problemas físicos a partir de sistemas ditos "análogos" têm revelado que problemas complexos podem eventualmente ser tratados a partir de um tratamento mais simples que aqueles impostos pela relatividade geral ou pela mecânica quântica. Um exemplo, em outro contexto, pode ser visto no estudo de buracos negros acústicos [ [⿴囗], o que constitui a análise de certas propriedades desta entidade astrofísica utilizando ferramentas de matéria condensada usual, isto é, a física de buracos negros é tratada a partir das características hidrodinâmicas de um fluido, permitindo determinar com isso a formação dos horizontes de eventos e a emissão

\footnotetext{
${ }^{3}$ E-mail: adriano.oliveira@ifes.edu.br.

de radiação por parte do misterioso corpo celeste. Existem outros exemplos semelhantes em que, de maneira geral, a mecânica newtoniana fornece excelentes resultados em diversos segmentos da astrofísica e cosmologia, desde a teoria de formação de estruturas até modelos de matéria e energia escuras, tais como a teoria

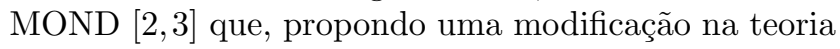
newtoniana, é capaz de resolver o problema da curva de rotação das galáxias espirais sem ter que recorer ao artifício da matéria escura. Portanto, mesmo sem o status de uma teoria fundamental, o qual já possuiu, a mecânica newtoniana conserva ainda grandes e interessantes aplicações em cenários puramente teóricos e ligados a física contemporânea de ponta, além de todas as incontáveis aplicacões técnicas que estão intimamente ligadas ao nosso dia-a-dia.

O grau de acuidade de uma teoria física é medido sob seu teste em determinado experimento. Este fornece três caminhos: ou a teoria não descreve e nem se adapta ao modelo proposto, ou ela precisa ser modificada com a inserção de novos e, em geral, mais complexos termos em suas equações, o que leva a considerações menos idealizadas tais como termos de viscosidade, acoplamentos entre coordenadas, dentre diversos outros, 
ou ainda a teoria é plenamente satisfatória e descreve com precisão o sistema ao qual se propõe, algo que depende em muito do quão próximo à realidade deseja-se descrever um determinado modelo. Tendo o segundo destes caminhos em mente, propomos neste trabalho, seguindo uma abordagem newtoniana, o estudo de um sistema clássico com alto grau de acoplamento entre as coordenadas e forte dependência com as velocidades em algumas direções. Em geral forças dependentes da velocidade têm seu caráter atribuído à sistemas que dissipam ou ganham (menos comum) energia. Contudo pode-se observar que em corridas de automóveis, por exemplo, quando um carro entra no chamado vácuo do carro imediatamente a frente, a diferença de pressão entre a traseira do carro perseguidor e sua dianteira, o impulsiona para frente, o que caracteriza um ganho de energia. Esse fato está diretamente relacionado com a economia de combustível do carro perseguidor. Outro exemplo de ganho ou dissipação de energia, com forças dependentes da velocidade, pode ser obtido em sistemas envolvendo o oscilador harmônico (com termos que o fazem ganhar ou perder energia) cuja expressão é dada por

$$
\ddot{x} \pm \gamma \dot{x}+\omega_{0}^{2} x=0 .
$$

A variação da energia com o tempo é

$$
\frac{d E}{d t}=m \dot{x} \ddot{x}+m \omega_{0}^{2} x \dot{x}=m \dot{x}\left(\dot{x}+\omega_{0}^{2} x\right)=\mp m \gamma \dot{x}^{2},
$$

que é positivo ou negativo. Quando positivo tem-se uma solução correspondente ao exemplo do carro perseguidor acima mencionado. Entretanto, existem sistemas [⿴囗 que, mesmo contendo dependências deste tipo em suas forças associadas, tem ainda assim sua energia conservada, o que é um resultado curioso. Tal fato se repete neste trabalho com um conjunto de forças ainda mais complexas. O aparente excesso de parâmetros ajustáveis na segunda lei de Newton associada ao modelo visa, ao contrário do que sugere, estabelecer da maneira menos restritiva possível as situações em que comportamentos conservativos são encontrados. Além disso, observa-se que a conservação da energia, neste caso, pode ocorrer eixo a eixo, isto é, associando a cada força que participa do sistema o eixo no qual se encontra decomposta, é possível verificar que, mesmo individualmente, a energia se conserva. Estes resultados serão apresentados na seção seguinte e as considerações finais serão tratadas nas conclusões.

\section{Forças e energias}

Em coordenadas esféricas a força resultante atuando sobre um sistema tem sua forma geral descrita por uma relação do tipo

$$
\begin{array}{r}
\mathbf{F}_{R}=\mathbf{F}_{r}(r, \theta, \phi, \dot{r}, \dot{\theta}, \dot{\phi})+\mathbf{F}_{\theta}(r, \theta, \phi, \dot{r}, \dot{\theta}, \dot{\phi})+ \\
\mathbf{F}_{\phi}(r, \theta, \phi, \dot{r}, \dot{\theta}, \dot{\phi}) .
\end{array}
$$

Escolheu-se aqui utilizar coordenadas esféricas para tratar um caso particular onde o termo que depende explicitamente da velocidade está contido apenas em uma componente da força, na direção de $\hat{\phi}$. Ou seja, as outra duas componentes $\hat{r}$ e $\hat{\theta}$ não dependerão da velocidade. Em geral para que um sistema com três ou dois dimensões seja dissipativo basta que apenas uma das componentes da força seja dependente da velocidade, ou seja, mesmo que as outras componentes da força NÃO sejam dependentes da velocidade o sistema como um todo é não-conservativo. Pretende-se mostrar que mesmo com essa dependência específica da força o sistema poderá ser conservativo, o que contraria o caso geral, pois era de se esperar, a priori, que o sistema como um todo fosse não-conservativo.

Em grande parte dos casos, considera-se que a força não depende da velocidade, pelos motivos discutidos no parágrafo anterior e ainda porque, em muitos casos, inviabiliza um estudo analítico ou uma descrição completa do sistema a ser estudado. Isso ocorre pois, a inclusão de tais termos, causam um aumento na complexidade matemática e, além disso, podem inviabilizar, por exemplo, obtenção das soluções explícitas e não-numéricas para as equações de movimento e, ainda, dificulta (ou impossibilita) o entendimento do papel de determinada força no sistema. Uma proposta de forma para a força resultante sobre um sistema curiosa, por depender da velocidade e resultar em uma conservação de energia, é dada por (lembrando que $\dot{\phi}=\frac{d \phi}{d t}$ e $\dot{\rho}=\frac{d \rho}{d t}$ )

$$
\begin{array}{r}
F_{R}=\left[\frac{f_{r}(\rho)}{\sin \theta}+g_{r}\right] \hat{r}+\left[\frac{f_{\theta}(\rho)}{\cos \theta}+g_{\theta}\right] \hat{\theta}+ \\
{\left[\frac{\eta \dot{\phi}}{2} \frac{\gamma}{r^{2} \sin ^{2} \theta} \frac{d}{d t}(\rho)\right] \hat{\phi},}
\end{array}
$$

sendo $\rho=r \sin \theta$ a componente radial em coordenadas cilíndricas. Essa forma foi escolhida pois é um modelo muito particular onde a força depende da velocidade e - dependendo da escolha das funções: $g_{r}, g_{\theta}, f_{r}$ e $f_{\theta}$ - possibilita a obtenção de uma solução não numérica para as equações diferenciais que resultam na equação de movimento para a partícula submetida a tal força. As funções $g_{r}, g_{\theta}, f_{r}$ e $f_{\theta}$ são as forças que definirão as características dinâmicas do sistema físico o qual trataremos neste trabalho; duas são conservativas $\left(f_{r}\right.$ e $\left.f_{\theta}\right)$ e duas não conservativas $\left(g_{r}\right.$ e $\left.g_{\theta}\right)$. O potencial resultante da força dada pela Eq. (四) poderia ser empregada em estudos das trajetórias de sistemas físicos sobre a influência de potenciais gravitacionais newtonianos ou de Manev.

Note que o termo da componente $\hat{\phi}$, na Eq. (四), é quem carrega a dependência da velocidade ${ }^{\square}$ nessa direção, que é exatamente a mesma utilizada no trabalho em duas dimensões [四], mas com $\rho=r \sin \theta$. Ainda assim será obtida uma conservação de energia no sistema. Além disso, dividimos as forças nas direções $\hat{r}$ e $\hat{\theta}$

\footnotetext{
${ }^{1}$ De acordo com os livros didáticos, para que a energia se conserve essa força deve ser nula. Ver maiores detalhes nas Refs. [, $\left.\mathbf{[}\right]$.
} 
com suas contribuições conservativa e não-conservativa, $f_{i}$ e $g_{i}$ respectivamente, sendo o índice $i$ igual a $r$ ou $\theta$, serão definidas posteriormente possibilitando a generalização das forças. As constantes $\eta$ e $\gamma$ garantem a dimensão de força da contribuição associada a coordenada $\hat{\phi}$. O trabalho [四] tratou de um problema semelhante em que a força na direção $\hat{\phi}$ era exatamente a mesma que a proposta acima, substituindo-se $\rho$ por $r \sin \theta$. Aqui porém o grau de complexidade é aumentado devido a presença de mais uma força ortogonal. Vale relembrar as relações entre os versores em coordenadas esféricas e seus correspondentes cartesianos. Assim,

$$
\begin{array}{r}
\hat{r}=\sin \theta \cos \phi \hat{i}+\sin \theta \sin \phi \hat{j}+\cos \theta \hat{k}, \\
\hat{\theta}=\cos \theta \cos \phi \hat{i}+\cos \theta \sin \phi \hat{j}-\sin \theta \hat{k}, \\
\hat{\phi}=-\sin \phi \hat{i}+\cos \phi \hat{j} .
\end{array}
$$

e da posição $\mathbf{r}=r \hat{r}$, obtem-se a velocidade e a aceleração sendo dadas por

$$
\begin{aligned}
& \mathbf{v}=\dot{r} \hat{r}+r \dot{\theta} \hat{\theta}+r \sin \theta \dot{\phi} \hat{\phi}, \\
& \mathbf{a}=\hat{r}\left(\ddot{r}-r \dot{\phi}^{2}-r \sin ^{2} \theta \dot{\phi}^{2}\right)+ \\
& \hat{\theta}\left(r \ddot{\theta}+2 \dot{r} \dot{\theta}-r \sin \theta \cos \theta \dot{\phi}^{2}\right)+ \\
& \hat{\phi}(r \sin \theta \ddot{\phi}+2 \sin \theta \dot{r} \dot{\phi}+2 r \cos \theta \dot{\theta} \dot{\phi}) .
\end{aligned}
$$

Ao aplicarmos a segunda lei de Newton a componente $\hat{\phi}$ da força,

$$
F_{\phi}=\mu(r \sin \theta \ddot{\phi}+2 \sin \theta \dot{r} \dot{\phi}+2 r \cos \theta \dot{\theta} \dot{\phi}),
$$

e multiplicá-lo por $2 r^{3} \sin ^{3} \theta \dot{\phi}$,

$$
\begin{aligned}
& \frac{2 r^{3} \sin ^{3} \theta \dot{\phi} F_{\phi}}{\mu}=2 r^{3} \times \\
& \sin ^{3} \theta \dot{\phi}(r \sin \theta \ddot{\phi}+2 \sin \theta \dot{r} \dot{\phi}+2 r \cos \theta \dot{\theta} \dot{\phi}) .
\end{aligned}
$$

Note que o lado direito da Eq. (प⿴囗) é igual a $\frac{d}{d t}\left[r^{4} \sin ^{4} \theta \dot{\phi}^{2}\right]$, então

$$
\frac{d}{d t}\left[r^{4} \sin ^{4} \theta \dot{\phi}^{2}\right]=\frac{2 r^{3} \sin ^{3} \theta \dot{\phi} F_{\phi}}{\mu},
$$

onde $\mu$ é a massa do corpo em estudo ou a massa reduzida, localizada no centro de massa, de um sistema constituído por dois ou mais componentes massivos. Substituindo a força $F_{\phi}$ na equação acima e multiplicando-a por $p\left[r^{4} \sin ^{4} \theta \dot{\phi}^{2}\right]^{p-1}$, sendo $p$ um número arbitrário diferente da unidade. A inclusão do número $p$ é apenas para ampliar as possibilidades de escolha dos termos polinomiais que podem ser utilizados para multiplicar a equação em questão. Tal escolha não deve influenciar o resultado final, ou seja, o resultado não deve depender de $p$ como de fato ocorre. Desse modo, temos

$\frac{d}{d t}\left[\log \left(\frac{r^{4} \sin ^{4} \theta \dot{\phi}^{2}}{r_{0}^{4} \sin ^{4} \theta_{0} \dot{\phi}_{0}^{2}}\right)^{p}\right]=p \eta \gamma \frac{d}{d t}\left[\frac{1}{2 \mu r^{2} \sin ^{2} \theta}\right]$.

Acima, $r_{0}, \theta_{0}$ e $\dot{\phi}_{0}$ são constantes de integração. Integrando novamente e isolando a velocidade angular $\dot{\phi}$, obtemos

$$
\dot{\phi}^{2}=\frac{k^{2} \dot{\phi}_{0}^{2}\left(r_{0}^{4} \sin ^{4} \theta_{0}\right)}{r^{4} \sin ^{4} \theta} \exp \left(\frac{\eta \gamma}{2 \mu r^{2} \sin ^{2} \theta}\right),
$$

onde $k^{2}$ é uma nova constante de integração. O desaparecimento de $p$ deve-se à propriedade de $\log , a \log b=$ $\log b^{a}$. Talvez o comportamento da matéria que compõe os anéis de Saturno, por exemplo, devido às perdas e ganhos da velocidade angular (causada pelas sucessivas colisões entre as partículas que o compõe) poderiam corresponder a esse comportamento.

O passo seguinte consiste em aplicar a segunda lei de Newton à força atuante na direção de $\hat{\theta}$. Com isso,

$$
F_{\theta}=\mu a_{\theta}=\mu\left(r \ddot{\theta}+2 \dot{r} \dot{\theta}-r \sin \theta \cos \theta \dot{\phi}^{2}\right) .
$$

Substituindo a Eq. (एव) na Eq. (피), encontramos

$$
\begin{array}{r}
F_{\theta}=\mu\left[r \ddot{\theta}+2 \dot{r} \dot{\theta}-\cos \theta \frac{k^{2}\left(r_{0}^{4} \sin ^{4} \theta_{0} \phi_{0}^{2}\right)}{r^{3} \sin ^{3} \theta} \times\right. \\
\left.\exp \left(\frac{\eta \gamma}{2 \mu r^{2} \sin ^{2} \theta}\right)\right] .
\end{array}
$$

Com a aplicação da segunda lei de Newton na componente $r$ temos

$$
F_{r}=\mu a_{r}=\mu\left[\ddot{r}-r \dot{\theta}-r \sin ^{2} \theta \dot{\phi}^{2}\right] .
$$

Utilizando novamente a Eq. (ए2) temos

$$
\begin{array}{r}
F_{r}=\mu\left[\ddot{r}-r \dot{\theta}-\frac{k^{2}\left(r_{0}^{4} \sin ^{4} \theta_{0} \phi_{0}^{2}\right)}{r^{3} \sin ^{2} \theta} \times\right. \\
\left.\exp \left(\frac{\eta \gamma}{2 \mu r^{2} \sin ^{2} \theta}\right)\right] .
\end{array}
$$

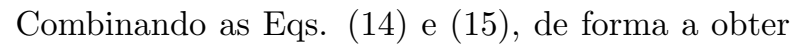
$F_{r} \sin \theta+F_{\theta} \cos \theta$, teremos

$$
\begin{aligned}
& \mu[\sin \theta(\ddot{r}-r \dot{\theta})+\cos \theta(r \ddot{\theta}+2 \dot{r} \dot{\theta})- \\
& \left.\left(\sin ^{2} \theta+\cos ^{2} \theta\right) \frac{k^{2}\left(r_{0}^{4} \sin ^{4} \theta_{0} \phi_{0}^{2}\right)}{r^{3} \sin ^{3} \theta} \exp \left(\frac{\eta \gamma}{2 \mu r^{2} \sin ^{2} \theta}\right)\right]= \\
& F_{r} \sin \theta+F_{\theta} \cos \theta .
\end{aligned}
$$

Visto que $\frac{d^{2}}{d t^{2}}(r \sin \theta)=\sin \theta(\ddot{r}-r \dot{\theta})+$ $\cos \theta(r \ddot{\theta}+2 \dot{r} \dot{\theta})$ e $\sin ^{2} \theta+\cos ^{2} \theta=1$ então,

$\mu\left[\frac{d^{2}}{d t^{2}}(r \sin \theta)-\frac{k^{2}\left(r_{0}^{4} \sin ^{4} \theta_{0} \phi_{0}^{2}\right)}{r^{3} \sin ^{3} \theta} \exp \left(\frac{\eta \gamma}{2 \mu r^{2} \sin ^{2} \theta}\right)\right]=$ $F_{r} \sin \theta+F_{\theta} \cos \theta$. 
Já que $\rho=r \sin \theta$ então pode-se escrever a expressão acima em termos de $\rho$. Para isso vamos multiplica-la por $\dot{\rho}$ e substituir as componente das forças dadas pela Eq. (四), assim

$$
\begin{array}{r}
\mu\left[\dot{\rho} \ddot{\rho}-\dot{\rho} \frac{k^{2}\left(r_{0}^{4} \sin ^{4} \theta_{0} \phi_{0}^{2}\right)}{\rho^{3}} \exp \left(\frac{\eta \gamma}{2 \mu \rho^{2}}\right)\right]= \\
\dot{\rho} f_{r}(\rho)+\dot{\rho} f_{\theta}(\rho)+\dot{\rho} g_{r} \sin \theta+\dot{\rho} g_{\theta} \cos \theta .
\end{array}
$$

Para que o lado direito da Eq. (ㅁ]) tenha apenas termos ligados a forças conservativas vamos escolher um $g_{r}$ e $g_{\theta}$ de forma que

$$
g_{r} \sin \theta+g_{\theta} \cos \theta=0 .
$$

Então, assumindo a Eq. (미) temos

$$
\begin{array}{r}
\mu\left[\dot{\rho} \ddot{\rho}-\dot{\rho} \frac{k^{2}\left(r_{0}^{4} \sin ^{4} \theta_{0} \phi_{0}^{2}\right)}{\rho^{3}} \exp \left(\frac{\eta \gamma}{2 \mu \rho^{2}}\right)\right]= \\
\dot{\rho} f_{r}(\rho)+\dot{\rho} f_{\theta}(\rho) .
\end{array}
$$

Integrando a Eq. ([ष]), a partir da Ref. [0], obtemos uma parcela da energia total

$$
E_{\rho}=\frac{1}{2} \mu \dot{\rho}^{2}+\frac{L_{0}^{2}}{\eta \gamma} \exp \left(\frac{\eta \gamma}{2 \mu \rho^{2}}\right)+U_{r}(\rho)+U_{\theta}(\rho)
$$

onde $L_{0}^{2}=k^{2}\left(r_{0}^{4} \sin ^{4} \theta_{0}{\dot{\phi_{0}}}^{2}\right)$ tem dimensão de momento angular ao quadrado e os termos $U_{r}$ e $U_{\theta}$ são as energias potenciais relacionados com $f_{r}(\rho)$ e $f_{\theta}(\rho)$. $\mathrm{O}$ fato de termos introduzido duas energias potenciais $U_{r}(\rho)$ e $U_{\theta}(\rho)$ não é de maneira alguma um complicador e nem invalida os cálculos pois $f_{r}(\rho)$ e $f_{\theta}(\rho)$ dependem somente de $\rho$. Além disso os lados direitos das equações das forças em $r$ e $\theta$ não dependem da velocidade em nenhuma direção. Isso não significa que estejamos dizendo que haja conservação de energia por eixos coordenados.

Obtemos portanto uma parcela da energia total do sistema que é dependente apenas da coordenada $\rho$ e de sua derivada em primeira ordem. Mais ainda, este termo de energia corresponde a uma quantidade conservada, o que é esperado tendo em vista a condição (ए7), a qual anula os termos não-conservativos.

Observemos agora o cálculo de $F_{r} \cos \theta-F_{\theta} \sin \theta$. Aqui, teremos após algumas manipulações algébricas

$$
\begin{array}{r}
\mu[\cos \theta(\ddot{r}-r \dot{\theta})-\sin \theta(r \ddot{\theta}+2 \dot{r} \dot{\theta})]= \\
F_{r} \cos \theta-F_{\theta} \sin \theta,
\end{array}
$$

mas note que $\frac{d^{2}}{d t^{2}}(r \cos \theta)=\cos \theta(\ddot{r}-r \dot{\theta})-$ $\sin \theta(r \ddot{\theta}+2 \dot{r} \dot{\theta})$. Como $z=r \cos \theta$, temos

$$
\mu \ddot{z}=F_{r} \cos \theta-F_{\theta} \sin \theta .
$$

Substituindo as componentes $F_{r}$ e $F_{\theta}$ fornecidas pela Eq. (田), podemos escrever

$\mu \ddot{z}=f_{r}(\rho) \cot \theta-f_{\theta}(\rho) \tan \theta+\left(\cos \theta g_{r}-\sin \theta g_{\theta}\right)$.
Da condição ([7), ou seja,

$$
g_{\theta}=-\frac{\sin \theta}{\cos \theta} g_{r}
$$

temos

$$
\mu \ddot{z}=f_{r}(\rho) \cot \theta-f_{\theta}(\rho) \tan \theta+\frac{g_{r}}{\cos \theta},
$$

onde foi utilizado $\sin ^{2} \theta+\cos ^{2} \theta=1$. Note que a equação acima exibe um termo ligado a forças nãoconservativas. É importante salientar ainda que $f_{r}$, $f_{\theta}$ e $g_{r}$ são até o momento arbitrários. Vamos tratar aqui um caso muito particular onde escolheremos as funções $f_{r}(\rho)$ e $f_{\theta}(\rho)$ como funções proporcionais e inversamente proporcionais a $\rho$, respectivamente. E a função $g_{r}$ é escolhida como uma composição de termos proporcionais e inversamente proporcionais a $r$ e a $\cos \theta$. Assim, a forma analítica destas três funções pode ser dada por

$$
\begin{gathered}
f_{r}(\rho)=-\xi_{r} \rho, \\
f_{\theta}(\rho)=-\frac{\xi_{\theta}}{\rho}, \\
g_{r}=-\sum_{n=1}^{\infty} \frac{\lambda_{n}}{r^{n+1} \cos ^{n} \theta}-\sum_{n=2}^{\infty} \beta_{n} r^{n-1} \cos ^{n} \theta .
\end{gathered}
$$

Essa pode não ser a única escolha para os parâmetros que resulta numa conservação de energia. Contudo, é um bom exercício matemático que resulta em uma interpretação física interessante.

A forma escolhida para $g_{r}$, na Eq. ([2]), nos permite generalizar as possíveis soluções cuja energia será conservativa. O somatório infinito é apenas para generalizar a expressão. Na prática só serão diferentes de zero os coeficientes que representarem um modelo para um específico potencial físico. Esse argumento serve para todos os somatórios desse trabalho. Ou seja, para qualquer ordem de potência como escrita acima resultará numa conservação da energia. Vale salientar que tanto $g_{r}$ quanto $g_{\theta}$ são aqui definidas como uma mistura de coordenadas cilíndricas e esféricas e isso se faz necessário para que as "energias" associadas aos eixos $z$ e $a$ possam ser conservadas.

Com isso a Eq. (․․) assume a forma

$\mu \ddot{z}=\xi_{r} z+\frac{\xi_{\theta}}{z}-\sum_{n=0}^{\infty} \frac{\lambda_{n}}{r^{n+1} \cos ^{n+1} \theta}-\sum_{n=2}^{\infty} \beta_{n} r^{n-1} \cos ^{n-1} \theta$

Multiplicando por $\dot{z}$ e integrando, podemos escrever

$$
\begin{array}{r}
E_{z}=\frac{1}{2} \mu \dot{z}^{2}+\frac{1}{2} \xi_{r} z^{2}- \\
\xi_{\theta} \log \left(\frac{z}{z_{0}}\right)+\sum_{n=1}^{\infty} \frac{\lambda_{n}}{n z^{n}}+\sum_{n=1}^{\infty} \frac{\beta_{n} z^{n}}{n} .
\end{array}
$$


Finalmente, reescrevendo a Eq. (며) em termos das Eqs. (西, [2]), após a integração, temos

$$
\begin{array}{r}
E_{\rho}=\frac{1}{2} \mu \dot{\rho}^{2}+\frac{L_{0}^{2}}{\eta \gamma} \exp \left(\frac{\eta \gamma}{2 \mu \rho^{2}}\right)+ \\
\frac{1}{2} \xi_{r} \rho^{2}+\xi_{\theta} \log \frac{\rho}{\rho_{0}} .
\end{array}
$$

Note que a Eq. ([2]), assim como a Eq. ([区8), constitui também uma parcela da energia total do sistema dependente apenas da coordenada $z$ e de sua derivada em primeira ordem $\dot{z}$. Mais uma vez, assim como ocorrido

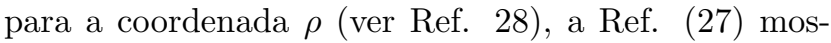
tra um legítimo comportamento de energia total (isto é, com um termo cinético e um potencial associado) e ainda conserva-se, o que é curioso tendo em vista que com as escolhas feitas, os termos não-conservativos não se anulam trivialmente. Isto sugere que a combinação dos termos escolhidos, a qual é uma condição muito particular, é tal que estes permitem a obtenção de uma quantidade conservada.

Para obter a parcela de energia dependente apenas da coordenada $\phi$ basta partir da Eq. (एव) e multiplicála por $\frac{\mu}{2} r^{2} \sin ^{2} \theta$. Deste modo

$$
\begin{array}{r}
\frac{\mu}{2} r^{2} \sin ^{2} \theta \dot{\phi}^{2}-\frac{\mu}{2} \frac{k^{2}\left(r_{0}^{4} \sin ^{4} \theta_{0} \phi_{0}^{2}\right)}{r^{2} \sin ^{2} \theta} \times \\
\exp \left(\frac{\eta \gamma}{2 \mu r^{2} \sin ^{2} \theta}\right)=0 \doteq E_{\phi} .
\end{array}
$$

A energia total será a soma das Eqs. (피), (피) e (ख्व) onde usamos também $\rho=r \sin \theta$ e $z=r \cos \theta$. Então

$$
\begin{aligned}
& E=E_{z}+E_{\rho}+E_{\phi}, \\
& E=\frac{1}{2} \mu\left(\dot{r}^{2}+r^{2} \dot{\theta}^{2}+r^{2} \sin ^{2} \theta \dot{\phi}^{2}\right)+ \\
& \frac{L_{0}^{2}}{\eta \gamma} \exp \left(\frac{\eta \gamma}{2 \mu r^{2} \sin ^{2} \theta}\right)+\frac{1}{2} \xi_{r} r^{2}+ \\
& \sum_{n=1}^{\infty} \frac{\lambda_{n}}{n r^{n} \cos ^{n} \theta}+\sum_{n=1}^{\infty} \frac{\beta_{n} r^{n} \cos ^{n} \theta}{n}+ \\
& \xi_{\theta} \log \left(\frac{\tan \theta}{\tan \theta_{0}}\right)-\frac{\mu}{2} \frac{k^{2}\left(r_{0}^{4} \sin ^{4} \theta_{0} \phi_{0}^{2}\right)}{r^{2} \sin ^{2} \theta} \times \\
& \exp \left(\frac{\eta \gamma}{2 \mu r^{2} \sin ^{2} \theta}\right)
\end{aligned}
$$

Há ainda outra possibilidade para a energia total. Ao invés de utilizar a condição (एШ) escolhemos uma nova condição a partir da Eq. (四). Essa escolha também resultará em um sistema conservativo. Para que isso ocorra temos

$$
\cos \theta g_{r}-\sin \theta g_{\theta}=0 .
$$

Com isso a Eq. (एव) fica modificada para

$$
g_{\theta}=\frac{\cos \theta}{\sin \theta} g_{r} .
$$

Isso pode ser interpretado como uma rotação de $\pi / 2$ em $\theta$ na Eq. (ש2), e somente nela. Note ainda que a forma da força e consequentemente da energia para os dois referênciais (o de antes e depois do giro de $\pi / 2$ no ângulo $\theta$ ) serão diferentes. Isso ocorre devido a forma matemática da força que atua no sistema ser diferente, o que resulta em expressões diferentes para a energia total.

Da Eq. (एव) com a condição (एव) e utilizando os valores de $f_{r}(\rho)$ e $f_{\theta}(\rho)$ fornecidos pelas Eqs. (एष) e (四), temos que

$$
\mu \ddot{z}=-\xi_{r} z+\frac{\xi_{\theta}}{z} .
$$

Multiplicando a equação acima por $\dot{z}$ e integrando, obtemos

$$
\frac{1}{2} \mu \dot{z}^{2}+\frac{1}{2} \xi_{r} z^{2}-\xi_{\theta} \log \left(\frac{z}{z_{c}}\right)=E_{z}
$$

Utilizando a Eq. ([6]) e substituindo $g_{\theta}=\cot \theta g_{r}$, ver Eq. (अ2), temos para a coordenada $\rho$

$$
\begin{aligned}
& \mu\left[\dot{\rho} \ddot{\rho}-\dot{\rho} \frac{k^{2}\left(r_{0}^{4} \sin ^{4} \theta_{0} \phi_{0}^{2}\right)}{\rho^{3}} \exp \left(\frac{\eta \gamma}{2 \mu \rho^{2}}\right)\right]= \\
& \dot{\rho} f_{r}(\rho)+\dot{\rho} f_{\theta}(\rho)+\dot{\rho} \frac{g_{r}}{\sin \theta}\left(\sin ^{2} \theta+\cos ^{2} \theta\right), \\
& \mu\left[\dot{\rho} \ddot{\rho}-\dot{\rho} \frac{k^{2}\left(r_{0}^{4} \sin ^{4} \theta_{0} \phi_{0}^{2}\right)}{\rho^{3}} \exp \left(\frac{\eta \gamma}{2 \mu \rho^{2}}\right)\right]= \\
& \dot{\rho} f_{r}(\rho)+\dot{\rho} f_{\theta}(\rho)+\dot{\rho} \frac{g_{r}}{\sin \theta},
\end{aligned}
$$

onde foi feito $\sin ^{2} \theta+\cos ^{2} \theta=1$. Propondo para $g_{r}$ a forma seguinte,

$$
g_{r}=\sum_{n=1}^{\infty} \frac{\lambda_{n}}{r^{n+1} \sin ^{n} \theta}-\sum_{n=2}^{\infty} \beta_{n} r^{n-1} \sin ^{n} \theta,
$$

ao substituir a Eq. (36) na Eq. (35) e integrarmos, teremos

$$
\begin{gathered}
E_{\rho}=\frac{1}{2} \mu \dot{\rho}^{2}+\frac{L_{o}^{2}}{\eta \gamma} \exp \left(\frac{\eta \gamma}{2 \mu \rho^{2}}\right)+\frac{1}{2} \xi_{r} \rho^{2}+ \\
\xi_{\theta} \log \left(\frac{\rho}{\rho_{c}}\right)+\sum_{n=1}^{\infty} \frac{\lambda_{n}}{n \rho^{n}}+\sum_{n=1, n \neq 2}^{\infty} \frac{\beta_{n} \rho^{n}}{n} .
\end{gathered}
$$

Portanto, neste caso, a energia total do sistema é a

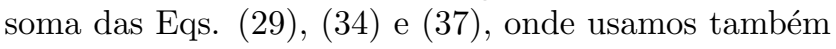
$\rho=r \sin \theta$ e $z=r \cos \theta$ ou seja

$$
\begin{aligned}
& E=E_{\rho}+E_{z}+E_{\phi}, \\
& E=\frac{1}{2} \mu\left(\dot{r}^{2}+r^{2} \dot{\theta}^{2}+r^{2} \sin ^{2} \theta \dot{\phi}^{2}\right)+ \\
& \frac{L_{0}^{2}}{\eta \gamma} \exp \left(\frac{\eta \gamma}{2 \mu r^{2} \sin ^{2} \theta}\right)+\frac{1}{2} \xi_{r} r^{2}+\xi_{\theta} \log \left(\frac{\tan \theta}{\tan \theta_{0}}\right)+ \\
& \sum_{n=1}^{\infty} \frac{\lambda_{n}}{n r^{n} \sin ^{n} \theta}+\sum_{n=1, n \neq 2}^{\infty} \frac{\beta_{n} r^{n} \sin ^{n} \theta}{n}- \\
& \frac{\mu}{2} \frac{k^{2}\left(r_{0}^{4} \sin ^{4} \theta_{0} \phi_{0}^{2}\right)}{r^{2} \sin ^{2} \theta} \exp \left(\frac{\eta \gamma}{2 \mu r^{2} \sin ^{2} \theta}\right) .
\end{aligned}
$$


E novamente note que a energia total é conservada como um todo mas também eixo a eixo.

\section{Conclusões}

Neste trabalho uma abordagem newtoniana para descrição de um sistema com alto grau de acoplamento entre forças em diferentes coordenadas foi proposta. Apesar da presença de uma força dependente da velocidade, o que confere a sistemas, em geral, características dissipativas, é possível mostrar analiticamente que a energia é conservada e, curiosamente, a energia total pôde ser separada em parcelas referentes a cada direção ortogonal $(\hat{\rho}, \hat{\theta}$ e $\hat{z})$ isto é, somente sob escolhas profundamente particulares das forças envolvidas pode ser possível escrever a energia total de um sistema como uma soma de energias conservadas para cada direção ortogonal sem comprometer, entretanto, a validade do teorema de conservação de energia. Enfatizamos mais uma vez que a especificidade deste resultado está relacionada às forças pouco convencionais analisadas de modo que esta pseudo-conservação de energia "por eixos" não constitui de maneira alguma uma generalização e muito menos reformula os resultados da teoria newtoniana largamente conhecida.

\section{Agradecimentos}

Os autores desejam agradecer aos árbitros da RBEF pelas correções e sugestões que enriqueceram muito esse artigo. As discussões acerca dos cálculos e conceitos foram de grande valia para os autores. A.M. Oliveira gostaria de agradecer à FAPES pelo apoio financeiro.

\section{Referências}

[1] W.G. Unruh, Phys. Rev. Lett. 46, xxx (1981).

[2] M. Milgrom, Astrophys. J. 270, 365 (1983); Astrophys. J. 270, 371 (1983); Astrophys. J. 270, 384 (1983)

[3] H.E.S. Velten, Revista Brasileira de Ensino de Física 30, $\operatorname{xxx}(2008)$.

[4] R.V. Sampaio, R. Fracalossi e A.M. Oliveira, Revista Brasileira de Ensino de Física 33, 1306 (2011).

[5] S.T. Thornton and J.B. Marion, Classical Dynamics of Particles and Systems (Brooks Cole, 2003), $5^{\mathrm{a}}$ ed.

[6] Kazunori Watari, Mecânica Clássica (Livraria da Física, São Paulo, 2003), $1^{\mathrm{a}}$ ed, v. 2. 